\title{
Modeling and Analysis of Structures with Negative Poisson's Ratio
}

\author{
P Siva Naga Sree M.E \\ Faculty, Department of Mechanical Engineering \\ D.M.S SVH College of Engineering \\ Machilipatnam, Krishna district, \\ Andhra Pradesh, India.
}

\author{
G. Sree Ram Charan Teja, Ch. Arun Sai Krishna, \\ B. Kanaka Durga Rao, A. Aditya Aravind. \\ B. Lakshamana Rao \\ Department of Mechanical Engineering \\ DMSSVH College of Engineering \\ Machilipatnam, Krishna district, \\ Andhra Pradesh, India.
}

\begin{abstract}
Auxetic structures are the structures with negative poisons ratio and display the peculiar behaviour of lateral expansion when they are stretched which is different from conventional structures. They get equal and opposing densification when compressed. Light weight, high strength, impact damping capabilities, and stiffness offers potential applications in the field of aerospace, automobile crash box, implantable devices, military protection equipment, suspension mount, intelligent actuators. The present work is focused on analysing the directional Auxeticity of tetra chiral Materials through design and analysis. The practical predictions about the global elastic properties of auxetic structures, such as re-entrant hexagonal cell, re-entrant star, re-entrant triangular and lozenge square structures are obtained with parametric 3D NX.11.0 and Ansys 19.2. A fused deposition Modelling in technique is used build prototypes of Auxetic structure. The analysis of the structures clearly established the auxetic behaviour of all the considered model's lateral expansion.
\end{abstract}

Keywords-Auxeticstructures,Negativepoissons ratio , Ansys, lateral expansion, Strain,Finite element analysis, NX 11.0.

\section{INTRODUCTION}

The terminology of" auxetic" was used by professor Ken Evans of the university of Exeter. One of the first artificially produced auxetic materials, the RFS structure (diamond-fold structure), was invented in 1978 by the Berlin researcher [1].Pietsch although did not use the term auxetics, he describes for the first time the underlying lever mechanism and its lateral expansion, therefore considered him as the inventor of the auxetic net. The earliest published example of a material with negative Poisson's constant is due to A. G. Polyakov in 1985[2],"Determination of the average characteristics of elastic frameworks"; the next auxetic material was came to prominence in Science in 1987, entitled foam structures with a Negative Poisson's Ratio by R.S. Lakes from the university viscos in Madison. The use of the word auxetic to refer to this property probably began in 1991[3]. Designs of composites with inverted hexagonal periodicity cell (auxetic hexagon), possessing negative Poisson ratios, were published in 1985.

Auxetic structures are efficient cellular materials that can absorb blast or impact energy through plastic deformation, thus protecting the structure. They are developing sacrificial solutions with light weight, high specific strength, high specific toughness and excellent energy dissipating properties, due to its negative Poison's ratio nature. The use of auxetic and non-auxetic panels in blast resistant structures had been employed by developers. Nonetheless, implementation of those energy dissipaters, explicitly as a uniaxial passive damper is restrained to limited studies, which highlight the potential need for further explorations.[4]when a material is stretched there is normally an accompanying reduction in width.

A measure of this dimensional change can be identified by Poisson's ratio, $v=-$ Lat. strain /long. strain, where long. strain and Lat. strain means strain in longitudinal direction and strain in lateral direction when material is compressed or stretched in axial direction.

Auxetic structures are those with a negative Poisson's ratio and display the unexpected property of lateral expansion when stretched, as well as equal and opposing densification when compressed. They expand and shrink like this due to interconnected network. This unique property offers enhanced functionality in the property of structures. Compared to conventional structures, negative poisson's ratio (NPR) structures offers increased plain strain, tunable density, fracture resistance, shear modulus, acoustic response.

The NPR structures have been considered for a wide range of smart and functional devices, including smart antennas, stretchable sensors, protective equipment and in many other applications from the fields of nanotechnology and biomedicine to defense and aerospace.Despite these advantages, they got difficulties in manufacturing due to complex design and limit their applications. For many reports they are focused on theoretical and material response. Different types of NPR structures also have been developed. These are categorized into several types such as 'chiral', 'reentrant', 'rotating' and honeycomb, and others. They found that $2 \mathrm{D}$ chiral and 3D geometries have highest negative poisons ratio and 3D pyramid has lowest.

The design or modelling of auxetic structures can be done by mechanical software's like NX, CAD, CREO, CATIA, solid works. In this project, we selected NX 11.0 [5] (Siemens plm solutions) as our design software. The analysis of these structures is done by Ansys 19.2.

\section{DESIGN AND ANALYSIS}

The Auxetic effect of four different geometric structures including Re-Entrant Hexagon (Evans), Arrow Head (Triangular), Re-Entrant Star (4-N), Lozenge Square were examined.[8] The geometries of each structure are shown in figures 1,2,3 and 4. 
Finite Element Analysis [11][12] with tensile loading conditions was performed on all these geometric structures using Ansys package version 19.2. For each design top surface was fixed to a rigid structure and the top surface was pulled with various input forces to calculate elongation in axial direction and transverse deformation. The characteristic strut ratio $\mathrm{h} / \mathrm{l}$ and re-entrant angle have significant effects on the properties of auxetic structures.

The following all geometries are modelled using NX 11.0.

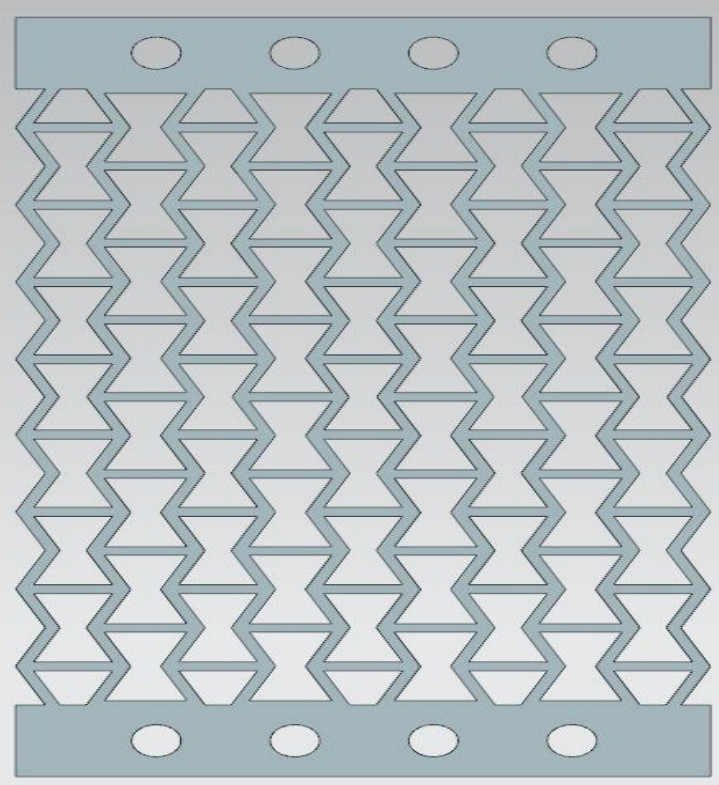

Fig. 1 Geometric model of Re-Entrant Evans structure The dimensions are $112.8 \times 57.2 \times 10$

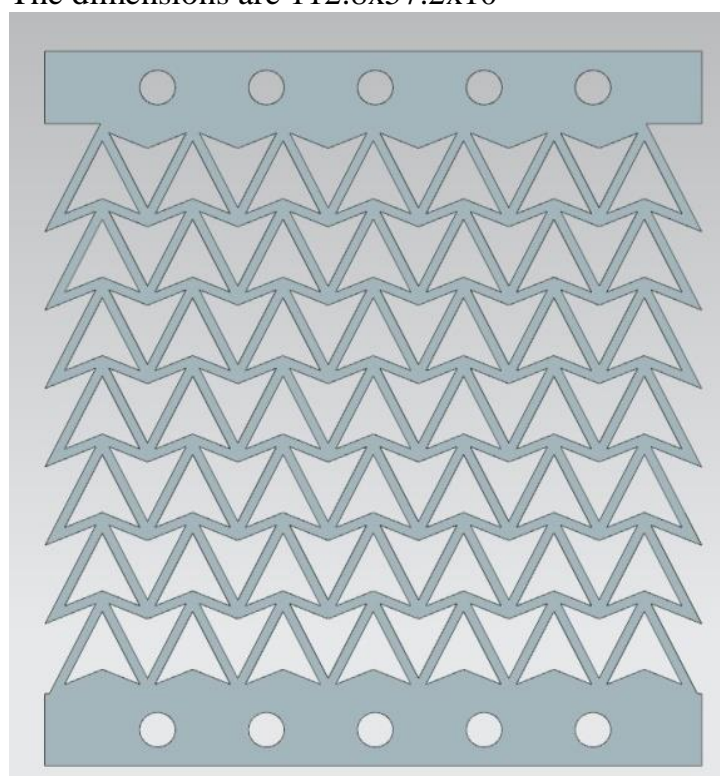

Fig. 2 Geometric model of Arrowhead structure

The dimensions are $98.8 \times 82.8 \times 10$.

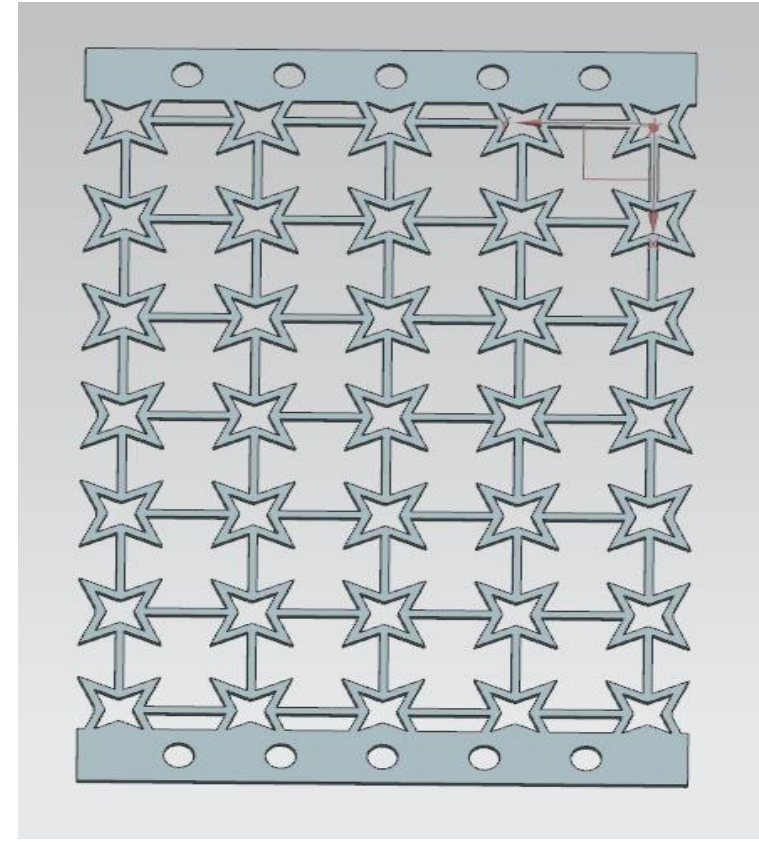

Fig. 3 Geometric model of STAR 4-N structure

The dimensions are 142.4 X 94.3x 10 .

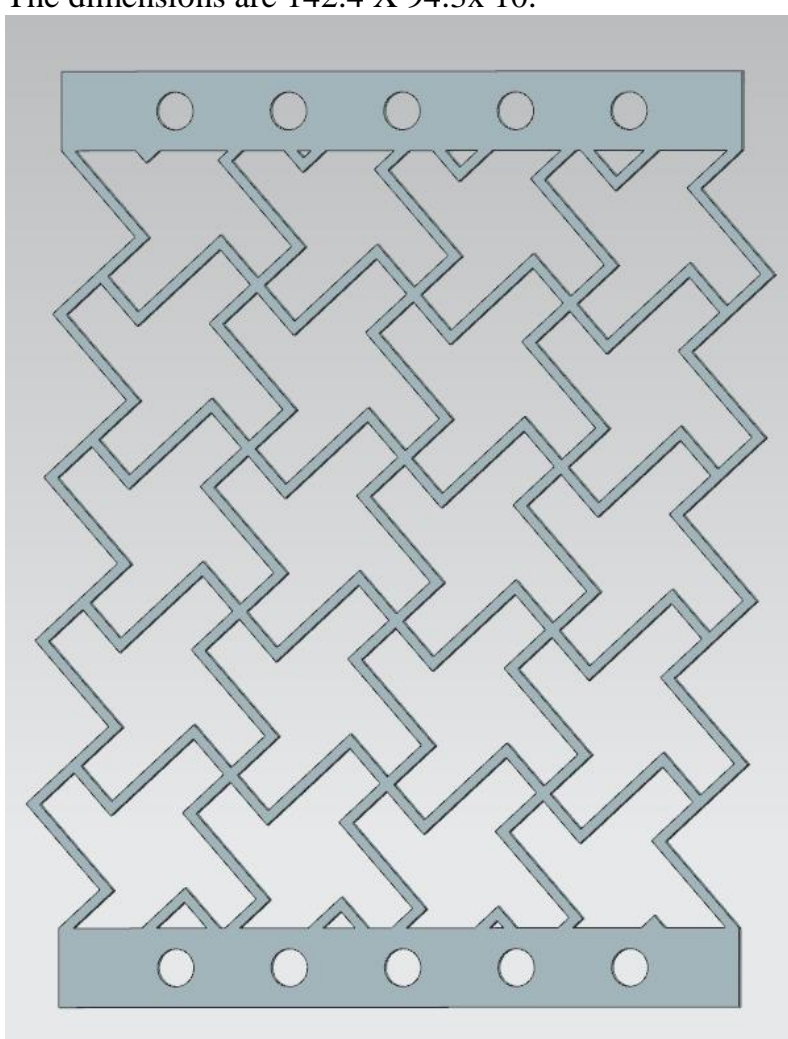

Fig. 4 Geometric model of LOZENGE SQUARE structure The dimensions are 118.1 X 89.4 X10.

Note: - All dimensions are in 'mm'.

\section{RESULTS AND DISCUSSIONS}

The four structures re-entrant Evans, arrowhead, reentrant star, lozenge square expands in the transverse direction as predicted and are shown in Fig 5,6,7 and 8. This confirms the auxetic behavior of the geometries.

The elongations of model-1 are as follows:

Length $X=6.72 \mathrm{e}-002 \mathrm{~m}$

Length $\mathrm{Y}=0.11281 \mathrm{~m}$ 
Length $\mathrm{Z}=1$. e- $002 \mathrm{~m}$

The poisons ratio of re-entrant Evans is -1.88

The elongations of model-2 are as follows:

Length $\mathrm{X}=8.8115 \mathrm{e}-002 \mathrm{~m}$

Length $\mathrm{Y}=9.877 \mathrm{e}-002 \mathrm{~m}$

Length $\mathrm{Z}=1 . \mathrm{e}-002 \mathrm{~m}$

The poisons ratio of arrowhead is -1.12

The elongations of model-3 are as follows:

Length $\mathrm{X}=0.14242 \mathrm{~m}$

Length $Y=9.433 \mathrm{e}-002 \mathrm{~m}$

Length $\mathrm{Z}=1$. e- $002 \mathrm{~m}$

The poisons ratio of re-entrant star is -0.6623

The elongations of model-4 are as follows:

Length $\mathrm{X}=9.5741 \mathrm{e}-002 \mathrm{~m}$

Length $\mathrm{Y}=0.12306 \mathrm{~m}$

Length $Z=1$. e- $002 \mathrm{~m}$

The poisons ratio of lozenge square is -1.367

Fig 5 Deformation of Evans structure at a load of 30N.

Page 1 of 1

\section{Directional Deformation}

Subject:
Author:
Prepared For:
Date $\quad$ Saturday, March 7, 2020
Comments:

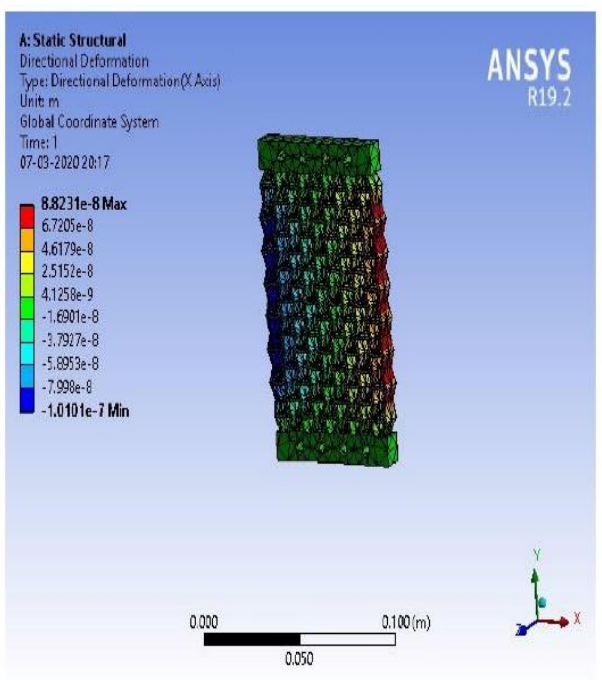

file:":C.Progran\%20Files ANSYS\%20Inciv192iaisol:DesignSpace:DSPagesihtunl:P... 07-03-2020
Fig 6 Deformation of Arrowhead structure at a load of $30 \mathrm{~N}$.

Page 1 of 1

\section{Directional Deformation}

Subject:

Author:

Prepared For

Date

Saturday, March 7, 2020

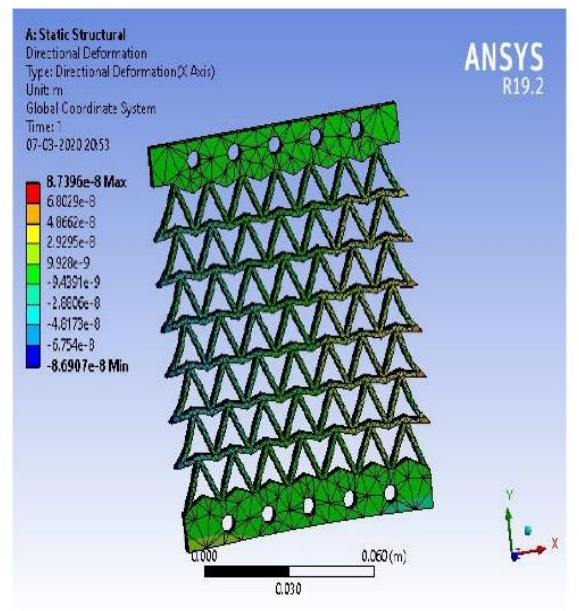

file: C: Program\%20Files $A$ NSYS\%20Inciv192:aisol:DesignSpace:DSPageshtuml:P... 07-03-2020

Table \#1 The deformation values in all three directions of four structures.

\begin{tabular}{|l|l|l|l|l|}
\hline SL.NO & STRUCTURE & X-DIRECION & Y-DIRECTION & Z-DIRECTION \\
\hline 1. & RE-ENTRANTEVANS & $6.72 \mathrm{e}-002 \mathrm{~m}$ & $0.11281 \mathrm{~m}$ & $1 . \mathrm{e}-002$ \\
\hline 2. & ARROWHEAD & $8.8115 \mathrm{e}-002 \mathrm{~m}$ & $9.877 \mathrm{e}-002 \mathrm{~m}$ & $1 . \mathrm{e}-002 \mathrm{~m}$ \\
\hline 3. & RE-ENTRANTSTAR & $0.14242 \mathrm{~m}$ & $9.433 \mathrm{e}-002 \mathrm{~m}$ & $1 . \mathrm{e}-002 \mathrm{~m}$ \\
\hline 4. & LOZENGESQUARE & $9.5741 \mathrm{e}-002 \mathrm{~m}$ & $0.12306 \mathrm{~m}$ & $1 . \mathrm{e}-002 \mathrm{~m}$ \\
\hline
\end{tabular}


Fig.7 Deformation of star 4-N structure at a load of 30N. Page 1 of 1

\section{Directional Deformation}

\begin{tabular}{ll}
\hline Subject: & \\
Author: & \\
Prepared For: & \\
Date & Sunday, March 8,2020 \\
Comments: &
\end{tabular}

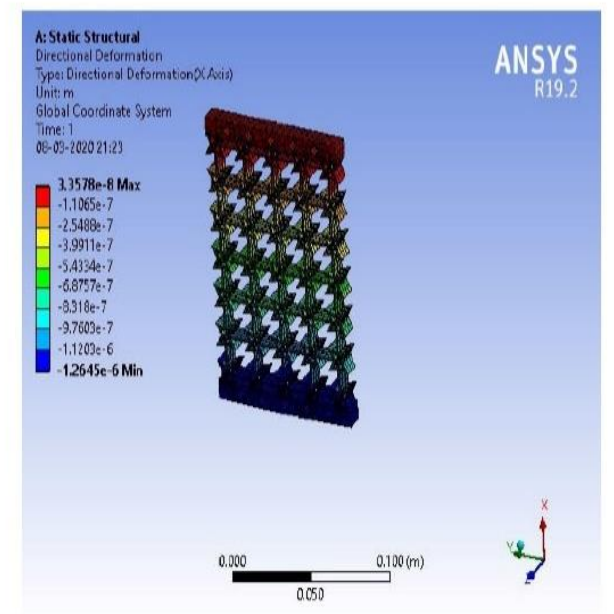

file://C. Program\%20Files/ANSYS"/n20Inc/v192/aisoliDesignSpace:DSPages/ittml/P... 08-03-2020

Table \#2 The poisons ratio of four auxetic structures

\begin{tabular}{|l|l|l|}
\hline SL NO: & STRUCTURE & POISSONS RATIO \\
\hline 1. & RE-ENTRANTEVANS & -1.8 \\
\hline 2. & ARROWHEAD & -1.12 \\
\hline 3. & RE-ENTRANTSTAR & -0.66 \\
\hline 4. & LOZENGE SQUARE & -1.36 \\
\hline
\end{tabular}

Fig.8 Deformation lozenge square structure at a load of $30 \mathrm{~N}$.

\section{Directional Deformation}

Subject:
Author:
Prepared For:
Date $\quad$ Sunday, March B. 2020
Comments:

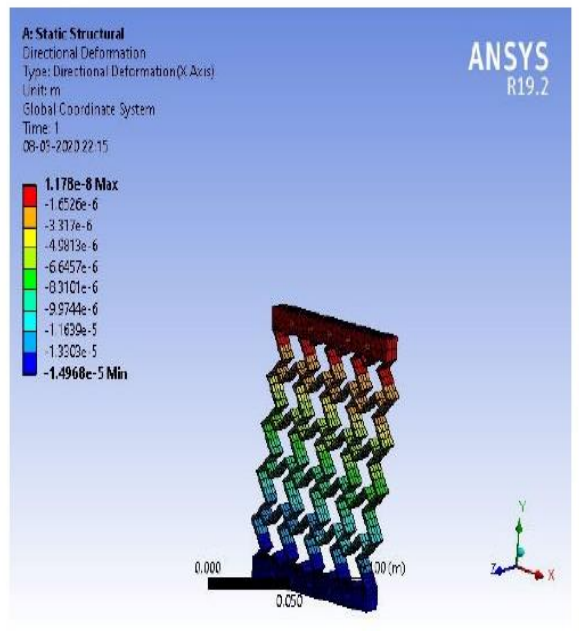

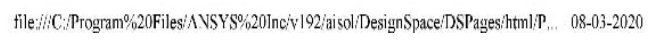

\section{Fabrication}

The materials generally used for preparing the auxetic structures are Poly lactic acid, Acrolytic butadiene styrene,Nylon, titanium, nickel, steels andall AM materials. Generally The auxetic structures are difficult to manufacture eventhough, the suitable technique for manufacturing auxetics is additive manufacturing techniques.[9] [10].In this work we fabricated the three models of our structures using PLA and ABS materials by 3D printing machine using FDM as shown in Fig 9 and 10. 


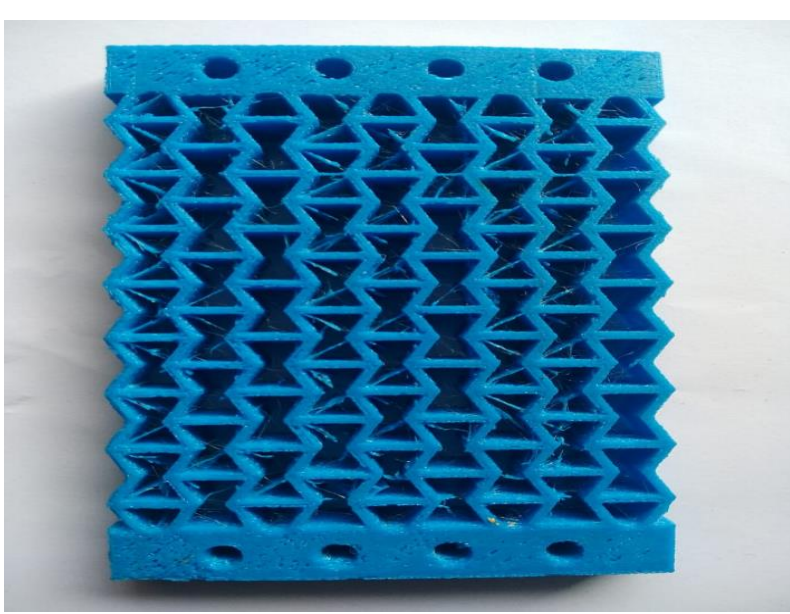

Fig. 9 fabricated model of EVANS structure using Fused deposition modelling

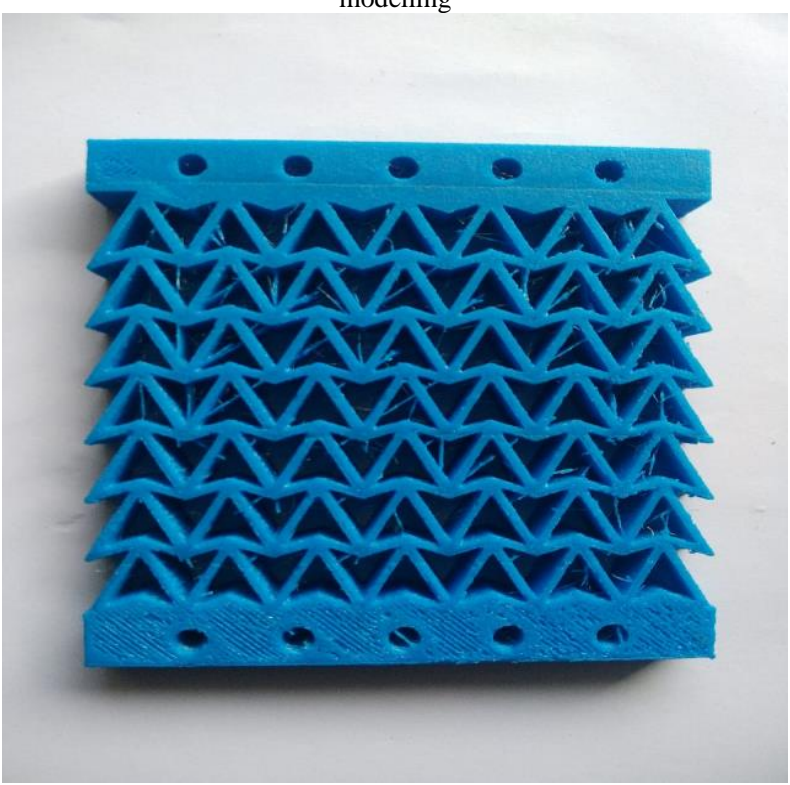

Fig.10 fabricated model of ARROWHEAD structure using Fused deposition modelling

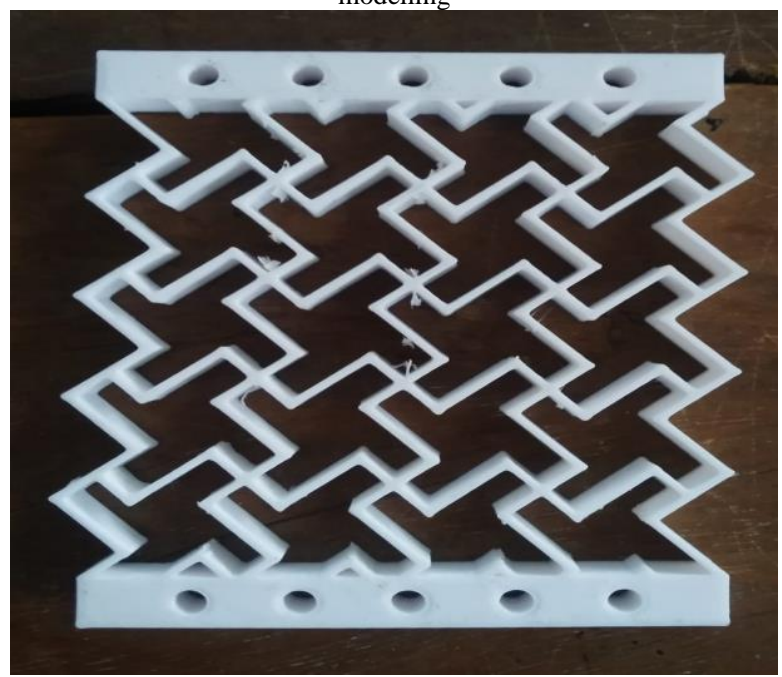

Fig 10 fabricated model of lozenge square structure using Fused deposition modelling

\section{CONCLUSIONS}

Auxetic materials have unique mechanical properties and excellent shock absorbing capability. In this work modelling and analysis of auxetic structures were done using 3D NX.11.0 and ANSYS 19.2. Out of all the four structures considered, Re-entrant Evans structure has the highest negative Poisson's ratio which implies the better auxetic behavior. The auxetic property can be obtained in products manufactured by additive manufacturing techniques. We have successfully fabricated the models in fused deposition modelling using PLA and ABS.

\section{REFERENCES}

[1] Evans, Ken (1991), "Auxetic polymers: a newrange of materials.", Endeavour, 15 (4): $\quad$ 170-174, doi:10.1016/01609327(91)90123 Kolpakov, A.G. (1985).

[2] Kolpakov, A.G. (1985). "Determination of the average characteristics of elastic frameworks". Journal of Applied Mathematics and Mechanics. 49 (6):

745. Bibcode:1985JApMM..49..739K. doi:10.1016/00218928(85)90011-5.

[3] Lakes, R.S. (27 February 1987), "Foam structures with a negative Poisson's ratio", Science, 235 (4792): 1038 40, Bibcode:1987Sci...235.1038L, doi:10.1126/science.235.4792.1038, PMID 17782252

[4] "The Development of a New Shock Absorbing Uniaxial Graded Auxetic Damper (UGAD)" Hasan Al-Rifaie * and Wojciech Sumelka Institute of Structural Engineering, Poznan University of Technology, 60-965 Poznan, Poland * Correspondence: hasan.alrifaie@put.poznan.pl

[5] Amruta Adasul, Himani Kadam. "Design and Analysis of Auxetic Honeycomb. Trends in Mechanical Engineering \& Technology "2019; 9(1): 46-58p

[6] Sheng Li a, Hany Hassanin b, Moataz M. Attallah a, *, Nicholas J.E. Adkins a, Khamis Essa

[7] "Review of Mechanics and Applications of Auxetic Structure" Mariam Mir, Murtaza Najabat Ali Javaria Sami and Umar Ansari

[8] "Comparative study of auxetic geometries by means of computer-aided design and engineering" Juan Carlos Alvarez Elipe and Andr' es D '1az Lantada Mechanical Engineering Department, Universidad Politecnica de Madrid, c/ Jos ' e Guti ' errez Abascal 2, ' 28006 Madrid, Spain

[9] "A novel layered topology of auxetic materials based on the tetra chiral honeycomb microstructure" Ferdinando Auricchio1, Andrea Bacigalupo2 *, Luigi Gambarotta3, Marco Lepidi3, Simone Morganti1, Francesca Vadalà3

[10] "Design and properties of 3D-printed chiral auxetic metamaterials by reconfigurable connections"

[11] "Finite Element Analysis of Three-Dimensional (3D) Auxetic Textile Composite under Compression "by Jifang Zeng and Hong $\mathrm{Hu}$

[12] "A finite element analysis of a 3D auxetic textile structure for composite reinforcement "Zhao yang Ge, Hong Hu and Yanping Liu 